\title{
Comparison of preoperative ultrasound guided fascia iliaca block versus femoral nerve block for proximal femur fractures before positioning for spinal anesthesia: an observational study
}

\author{
Meeta Gupta and Shaila Surendra Kamath \\ Department of Anesthesiology, Kasturba Medical College of Mangalore, Manipal Academy of Higher Education, Manipal, India
}

Received December 5, 2019

Revised January 23, 2020

Accepted January 28, 2020

\section{Correspondence}

Shaila Surendra Kamath

Department of Anesthesiology, Kasturba

Medical College of Mangalore, Manipal

Academy of Higher Education, 203 Light

House Hill Rd., Hampankatta, Manipal,

Karnataka 575001, India

Tel: $+91-824-2445858$

Fax: +91-824-2428183

E-mail: shailakamath@ymail.com

Previous presentation at conferences: The 43rd State Conference of ISA Kerala Chapter, 18-20th Oct. 2019, Thrissur, India.
Background: Severe pain associated with proximal femur fractures makes the positioning for regional anesthesia a challenge. Systemic administration of analgesics can have adverse effects. Individually, both the fascia iliaca block (FIB) and femoral nerve blocks (FNB) have been studied. However, there is little evidence comparing the two. The aim of this study was to compare the overall efficacy of the two blocks in patients with proximal femur fracture before positioning for spinal anesthesia. Methods: ASA (American Society of Anesthesiologists) class I, II, and III patients scheduled for elective and emergency surgery with the diagnosis of proximal femur fracture between October 2018 and June 2019 were included in the study. The patients were assigned to two groups by convenience nonprobability sampling of 35 each.

Results: Our study showed a reduction in visual analogue scale scores at 3, 4, and 5 minutes after administration of the FIB being $5.1 \pm 1.1,4.1 \pm 1.3$, and $2.8 \pm 0.8$, and those after the FNB as $4.4 \pm 1.1,3.3 \pm 1.1$, and $2.1 \pm 1.4$ with $P<0.05$, which was statistically significant. The mean first rescue analgesia time for the FIB was $7.1 \pm 2.1$ hours, while for the FNB it was $5.2 \pm 0.7$ hours. The $P$ value was less than 0.001 , which was significant.

Conclusions: Both ultrasound guided FNB and FIB techniques provide sufficient analgesia for patient's positioning before spinal anesthesia. However, the duration of postoperative analgesia provided by FIB was greater than that of the FNB.

Key Words: Analgesics; Anesthesia, Spinal; Bupivacaine; Fascia; Femoral Nerve; Fractures, Bone; Nerve Block; Pain Management; Ultrasonography.

\section{INTRODUCTION}

The fractures of the proximal femur, including the fracture of the neck of femur, intertrochanteric, and subtrochanteric fractures, are a common orthopedic emergency, especially in the geriatric population [1]. These fractures are extremely painful and associated with significant morbidity and mortality $[2,3]$. Regional anesthesia is preferred during surgery in these patients. However, the severe pain associated with these injuries makes appropriate positioning difficult for the regional anesthesia, thus altering their overall success rate [4]. Pain can be detrimental in this group of patients, as it causes tachycardia and hypertension, due to enhanced catecholamine release [5].

Opioids have been the main modality for conventional pain management, which are known to have serious (a) This is an open-access article distributed under the terms of the Creative Commons Attribution Non-Commercial License (http://creativecommons.org/licenses/by-nc/4.0/), which permits unrestricted non-commercial use, distribution, and reproduction in any medium, provided the original work is properly cited.

(c) The Korean Pain Society, 2020
Author contributions: Meeta Gupta: Writing/manuscript preparation; Shaila Surendra Kamath: Writing/manuscript preparation. 
systemic side effects. To overcome this problem, various nerve blocks have been tried. They not only help in proper patient positioning preoperatively by providing preoperative analgesia, but also give postoperative pain relief. Further, several studies which compare nerve blocks and intravenous opioids for better positioning, and for postoperative analgesia for fractured femur have proven the supremacy of nerve blocks [6].

In 1980, Sharrock [7] was the first to describe the fascia iliaca block (FIB). The femoral nerve block (FNB) was first illustrated by Winnie et al. [8] in 1973. Both the techniques aim to anesthetize the lateral femoral cutaneous nerve, femoral nerve, and obturator nerve by using an anterior thigh approach technique [9]. The use of ultrasonography in anesthesia has helped the anesthetist to see the nerve, needle, and the distribution of the drug, thereby enhancing the chances of a favourable outcome of the nerve block. Grade A recommendation for an ultrasound guided approach to the peripheral nerve blocks is supported by level Ib evidence for a shortened time for the onset of sensory block, decreased performance time, and lower drug doses [10].

Although individually both the blocks have been studied, there are only a handful of studies comparing both techniques under ultrasound guidance, for making patient positioning for spinal anesthesia easy.

Our study design was to compare the FIB with the FNB using ultrasound to assess the overall efficacy for pain relief during patient positioning for spinal anesthesia.

\section{MATERIALS AND METHODS}

\section{Patients}

After receiving approval from the Institutional Scientific and Ethics Committee of the Kasturba Medical College (IEC KMC MLR 09-18/335), seventy ASA (American Society of Anesthesiologists) class I, II, and III patients were included in the study, who were planned for elective and emergency surgery, with the diagnosis of proximal femur fracture between October 2018 and June 2019. As per the protocol, a detailed pre-anesthetic check-up and investigation was done. The study protocol was explained to the patients and written informed consent was obtained. The monitors connected were electrocardiogram, pulse oximetry, and non-invasive blood pressure, in the preoperative area. For drug and fluid administration, an intravenous cannula of suitable size was obtained.

The patients were divided into 2 groups by convenience non-probability sampling. Group FIB ( $\mathrm{n}=35$, those who received an FIB with $30 \mathrm{~mL}$ of $0.25 \%$ bupivacaine) and group FNB ( $\mathrm{n}=35$, those who received an FNB with $30 \mathrm{~mL}$ of $0.25 \%$ bupivacaine). Depending on the allotted group, patients were given the block under the guidance of ultrasound in the preoperative area by an experienced anesthesiologist with more than two years' experience.

\section{Technique}

The FNB was performed as follows: under ultrasound guidance (Venue 40 Ultrasound; GE Healthcare, Wauwatosa, WI), the femoral artery was identified, by keeping the linear probe (B mode, 8-18 MHz frequency) in an "in line plane" technique. The femoral nerve was recognized as a hyper echoic structure, which is triangular in shape and located lateral to femoral artery [11]. An insulated 22 $\mathrm{G}$ needle was inserted and positioned by the side of femoral artery. A small volume of local anesthetic was injected after locating the needle tip and a negative aspiration for blood. After the distribution of the local anesthetic was seen, the rest of the drug volume was injected.

The FIB was given in a similar fashion. Lateral to the femoral nerve, the psoas muscle was located. It is covered by the iliacus fascia, which is deep to the fascia lata. Therefore, after introducing the needle, a double pop was felt until the needle had reached a position between the fascia iliaca and psoas muscle. Similar to the technique stated above, $1 \mathrm{~mL}$ of the local anesthetic solution was injected, after negative aspiration for blood, and spread of the drug was seen under the fascia iliaca.

A $10 \mathrm{~cm}$ visual analogue scale (VAS) score was recorded before the block and immediately after the block at 1, 2, 3, $4,5,10$, and 15 minutes. The patients were made to sit for spinal anesthesia when the VAS score was less than 3 . The VAS score was measured immediately postoperatively and at intervals of 2 hours, for a period of 24 hours. If the VAS score was $>4$, rescue analgesia was given; an injection of paracetamol $10 \mathrm{mg} / \mathrm{kg}$ was given over a period of 10 to 15 minutes as an intravenous infusion. Postoperatively, first analgesic request time and the total dose of intravenous paracetamol was recorded.

Data was analysed by using a student unpaired $t$-test, student paired test, and chi-square test. $P$ values less than 0.05 were considered as significant. SPSS ver. 17.0 (SPSS Inc., Chicago, IL) was used to do the analysis.

\section{RESULTS}

The mean age in the FIB group was $61.9 \pm 16.7$ years while in the FNB group $64.7 \pm 13.6$ years. There was predominance of females in the FIB group (57.1\%) where as in the FNB group, the majority were males (51.4\%). The larger 
part of the patients in both the groups was ASA grade II (Table 1).

The values of the mean and standard deviation for VAS scores for the FIB and FNB groups at 3 minutes was 5.1 \pm 1.1 and $4.4 \pm 1.1$, respectively, with a $P$ value of 0.006 , which was statistically significant. At 4 and 5 minutes, they were found to be $4.1 \pm 1.3,3.3 \pm 1.1$ for the FIB group, and $2.8 \pm 0.8,2.1 \pm 1.4$ for the FNB group. The $P$ values at 4 and 5 minutes were 0.004 and 0.017 , respectively, which was also statistically significant, thus signifying that the onset of the FIB was delayed by 2 minutes in comparison to the FNB. However, both the blocks achieved clinically significant pain reduction after 5 minutes with a $P<0.05$ as shown in Fig. 1.

The mean values of the intra-operative and post-operative heart rate between the FIB group and FNB group at $0,5,10,15$ minutes after the block and every 2 hours till 24 hours after the surgery are presented in the Fig. 2 . The $P$ value relationship of the two groups was found to be statistical insignificant, indicating both the techniques affect the heart rate in a similar manner. There was no significant statistical variability in the mean arterial pressures intraoperatively and postoperatively after both the blocks as shown in Fig. 3. The mean first rescue analgesia time for the FIB group was $7.1 \pm 2.1$ hours, while for the FNB group was $5.2 \pm 0.7$ hours. A remarkably significant $P<0.001$ was obtained. Therefore, the period of analgesia given by the FIB was more than that by the FNB as shown in Fig. 4.

Table 1. Demographic Profile

\begin{tabular}{lcc}
\hline \multicolumn{1}{c}{ Variable } & $\begin{array}{c}\text { FIB group } \\
(\mathrm{n}=35)\end{array}$ & $\begin{array}{c}\text { FNB group } \\
(\mathrm{n}=35)\end{array}$ \\
\hline Age $(\mathrm{yr})$ & $61.9 \pm 16.7$ & $64.7 \pm 13.6$ \\
Male/Female & $15 / 20$ & $18 / 17$ \\
ASA grade I/II/III & $9 / 21 / 5$ & $6 / 16 / 13$ \\
\hline
\end{tabular}

Values are presented as mean \pm standard deviation or number only. FIB: fascia iliaca block, FNB: femoral nerve block, ASA: American Society of Anesthesiologists.
There was no deviation in the total analgesic demand in both the groups in the 24 hours period as shown in Fig. 5 .

\section{DISCUSSION}

Our study demonstrates that the onset of FNB was earlier than that of the FIB. A statistically significant difference was seen in the $P$ value at 3,4 , and 5 minutes after the block. However, both the groups achieved a clinically significant degree of analgesia at 5 minutes after the block. No remarkable dissimilarity was seen in the heart rate or mean arterial pressures intraoperatively or postoperatively between the groups. The mean duration of postoperative analgesia provided by the FIB was greater than the FNB, which was statistically significant.

Improved quality and accuracy of the nerve blocks was seen under ultrasound guidance as compared to the use of anatomical landmarks or a nerve stimulator [12]. Although studies have been done on the FNB and FIB individually proving the superiority of both to intravenous opioids, there is insufficient literature, at present, comparing these

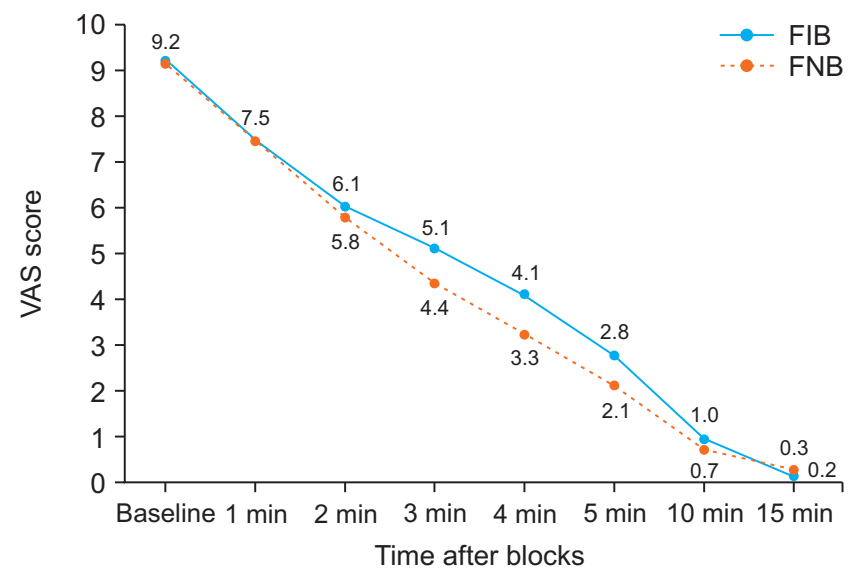

Fig. 1. Preoperative mean visual analogue scale (VAS) score. FIB: fascia iliaca block, FNB: femoral nerve block.

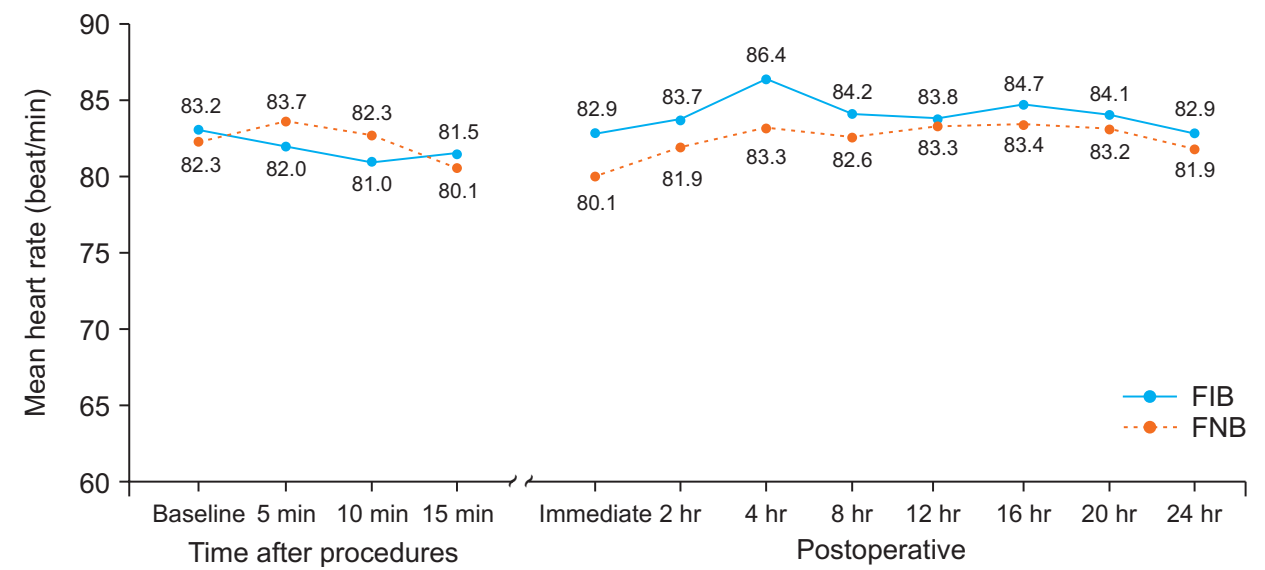

Korean J Pain 2020;33(2):138-143
Fig. 2. Intra operative and postoperative heart rate. FIB: fascia iliaca block, FNB: femoral nerve block. 


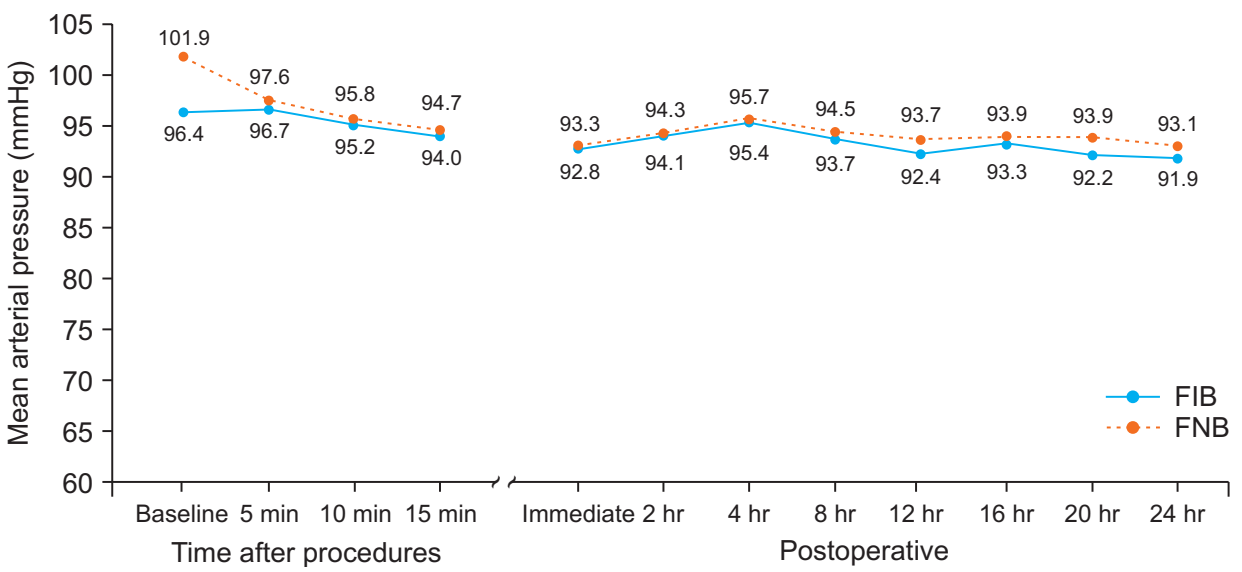

Fig. 3. Intra operative and postoperative mean arterial pressure. FIB: fascia iliaca block, FNB: femoral nerve block.

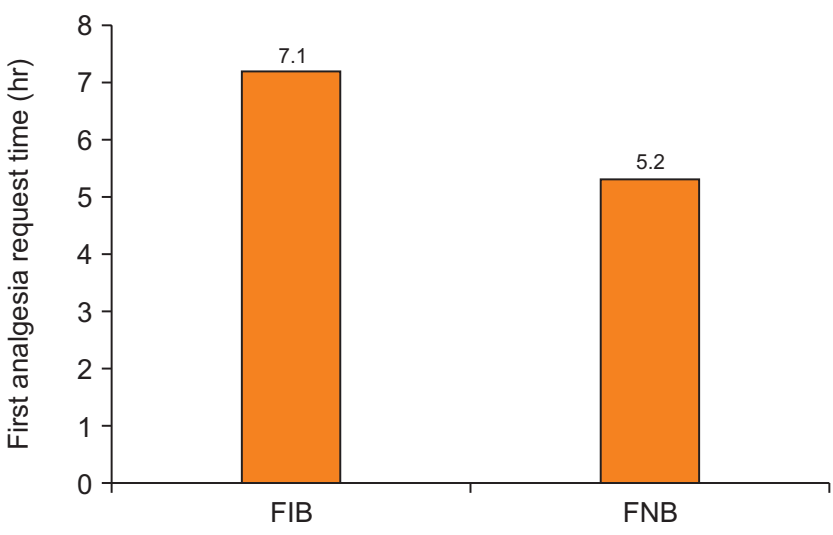

Fig. 4. First analgesia request time. FIB: fascia iliaca block, FNB: femoral nerve block.

blocks, using ultrasound assistance for pre-spinal anesthesia positioning and postoperative analgesia.

Yun et al. [13] did a study on elderly patients (62-88 yr) with fractures of the neck femur, concluding that the FIB, using $30 \mathrm{~mL}$ of $0.375 \%$ ropivacaine, was better than intravenous alfentanil, with the VAS score, as assessed 20 minutes after the block for positioning, being VAS score 2 for FIB versus 3.5 for the opioid group, with a highly significant $P=0.006$. Our study, using $30 \mathrm{~mL}$ of $0.25 \%$ bupivacaine, indicated a decrease in the VAS score at 5 minutes to $2.8 \pm 0.8$. This delayed onset of analgesia in the above study could be due to use of ropivacaine which has a delayed onset as compared to bupivacaine.

Williams et al. [14] and Groot et al. [15], in their respective studies, showed that FIB was better as compared to intravenous analgesics. Williams et al. [14] used $30 \mathrm{~mL}$ of $0.25 \%$ levobupivacaine, resulting in a reduction of VAS score to less than 3 in 15 minutes, and analgesia lasted up to 8 hours as compared to intravenous opioids, with a $P<$ 0.001 which was highly significant. This can be due to a slower onset but prolonged duration of action for levobupivacaine. Groot et al. [15] used $30 \mathrm{~mL}$ of $0.5 \%$ levobupivacaine to achieve adequate pain relief in $65 \%$ of the patients

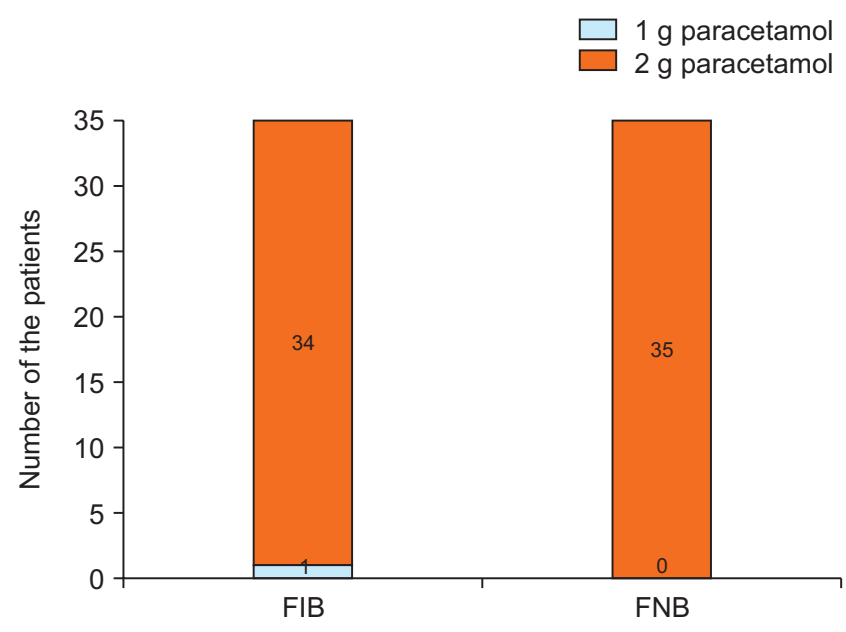

Fig. 5. Total amount of analgesia required in 24 hours. FIB: fascia iliaca block, FNB: femoral nerve block.

30 minutes after administration of the block, even when given by using the landmark technique. Sia et al. [6], in their study, concluded that the FNB given with the aid of a peripheral nerve stimulator, with $15 \mathrm{~mL}$ of $1.5 \%$ lignocaine, was successful at achieving a VAS score of $0.5 \pm 0.5$, 5 minutes after local anesthetic was dispensed, for spinal anesthesia positioning, as compared to an intravenous fentanyl group which had a VAS score of $3.3 \pm 1.4$. This rapid decline in VAS score could be due to the use of lignocaine which has rapid onset of action as compared to bupivacaine. Fletcher et al. [16] showed that the FNB, using 20 $\mathrm{mL}$ of $0.5 \%$ bupivacaine was a superior mode of analgesia to intravenous morphine. The 24-hour morphine dosage was less in the FNB group.

Callear et al. [17], in a non-comparative study, demonstrated that the FIB, using $30 \mathrm{~mL}$ of $0.25 \%$ bupivacaine, reduced pain considerably in 15 minutes after the block was given, and the analgesia lasted up to 8 hours, and also reduced the total analgesic requirement. Comparable results were shown by Kumar et al. [18] in their study, where they used $30 \mathrm{~mL}$ of $0.5 \%$ ropivacaine to give an FIB to relieve 
pain, before positioning for spinal anesthesia as assessed, 20 minutes after the block was given. The VAS score was reduced to 2.94 with a $P<0.01$, which has statistical significance. Ghimire et al. [19] demonstrated in their study that FIB when given, using $1.5 \%$ lignocaine with adrenaline up to a total volume $30 \mathrm{~mL}$, was more effective in reducing the VAS score $(1.0 \pm 1.1)$ than the FNB $(2.1 \pm 0.8)$, with $15 \mathrm{~mL}$ of the same drug and concentration, as seen 20 minutes after administering the block.

Our study showed a reduction in the VAS score at 3, 4, and 5 minutes after administration of the FIB being 5.1 $\pm 1.1 ; 4.1 \pm 1.3$; and $2.8 \pm 0.8$, respectively, and that of the FNB being $4.4 \pm 1.1 ; 3.3 \pm 1.1$, and $2.1 \pm 1.4$, respectively, with a $P<0.05$, which was statistically significant. However, the VAS score was less than 3 (the minimum required for patient positioning for spinal anesthesia in our study) after 5 minutes in both the groups. The difference could be attributed to unequal volumes of lignocaine in the study by Ghimire et al. [19] and the blind technique of the block using paresthesia. Capdevila et al. [20] compared the FIB and FNB using $15 \mathrm{~mL}$ of $2 \%$ lidocaine with 1:200,000 epinephrine and $15 \mathrm{~mL}$ of $0.5 \%$ bupivacaine, demonstrating the FIB being more beneficial in blocking both the lateral femoral cutaneous and femoral nerve in adults, simultaneously. This could account for a longer postoperative analgesia for the FIB, as seen in our study.

Our findings were comparable to those of Cooper et al. [11], in that ultrasound guided FNB and FIB have a similar success rate. However, previous studies by Newman et al. [21] had shown a superiority of the FNB over the FIB when performed using a nerve stimulator. This dissimilarity to our study could be due to a superior technique (ultrasound guided over nerve stimulation guided) which made both the blocks equally efficacious.

In our study, the five-minute interval after the block was sufficient to provide adequate analgesia to proceed to spinal anesthesia in all the patients. Therefore, the choice of the technique can be guided by the experience of the anaesthetist. The FIB has been considered relatively safe and easy to perform in various studies and can be preferred over the FNB $[15,17,18]$. In addition, the duration of analgesia provided postoperatively was also longer with the FIB than the FNB in our study.

Bupivacaine has been used previously for analgesia in both the FNB and FIB $[4,9,14,17,21]$. With an onset of action between 2 and 5 minutes, the maximum plasma levels are reached in 15-30 minutes [22]. Administering such a large quantity of local anaesthetic in so close to the femoral vessel can have some adverse effects. Therefore, it is imperative that only adequately trained personnel should administer such blocks. Also, proper monitoring and resuscitation facilities should be ready.
All the outcome variables except for duration of postoperative analgesia were comparable. The FIB was found to give a longer duration of post-procedure analgesia. This could be attributed to the prolonged stay of the drug under the fascia iliaca. Even though the FIB gives a longer period of analgesia than the FNB by approximately 2 hours, the total amount of analgesia required in 24 hours was almost the same.

Thus, we conclude that both the FIB and the FNB are equally efficacious for ease of positioning for spinal anesthesia in patients with proximal femur fractures. But the duration of postoperative analgesia was longer in the FIB.

\section{ACKNOWLEDGMENTS}

We would like to thank Kasturba Medical College, Manipal Academy of Higher Education, Manipal, for permitting us to use clinical material for conduct of this study.

\section{CONFLICT OF INTEREST}

No potential conflict of interest relevant to this article was reported.

\section{FUNDING}

No funding to declare.

\section{ORCID}

Meeta Gupta, https://orcid.org/0000-0001-9681-0646

Shaila Surendra Kamath, https://orcid.org/0000-0002-6824-5122

\section{REFERENCES}

1. Fujihara Y, Fukunishi S, Nishio S, Miura J, Koyanagi S, Yoshiya S. Fascia iliaca compartment block: its efficacy in pain control for patients with proximal femoral fracture. J Orthop Sci 2013; 18: 793-7.

2. Gupta N, Jain P, Kapadia M, Sanwatsarkar S, Saxena D, Maheshwari M. The effect of $0.25 \%$ levobupivacaine and $0.25 \%$ ropivacaine in fascia iliaca block in elderly patients with fracture femur given before positioning for subarachnoid block. Int J Res Med Sci 2018; 6: 191-4.

3. Rowlands M, Walt GV, Bradley J, Mannings A, Armstrong S, Bedforth $\mathrm{N}$, et al. Femoral nerve block intervention in neck of femur fracture (FINOF): a randomised controlled trial. 
BMJ Open 2018; 8: e019650.

4. Iamaroon A, Raksakietisak M, Halilamien P, Hongsawad J, Boonsararuxsapong K. Femoral nerve block versus fentanyl: analgesia for positioning patients with fractured femur. Local Reg Anesth 2010; 3: 21-6.

5. Yezierski RP. The effects of age on pain sensitivity: preclinical studies. Pain Med 2012; 13 Suppl 2: S27-36.

6. Sia S, Pelusio F, Barbagli R, Rivituso C. Analgesia before performing a spinal block in the sitting position in patients with femoral shaft fracture: a comparison between femoral nerve block and intravenous fentanyl. Anesth Analg 2004; 99: 12214.

7. Sharrock NE. Inadvertent "3-in-1 block" following injection of the lateral cutaneous nerve of the thigh. Anesth Analg 1980; 59: 887-8.

8. Winnie AP, Ramamurthy S, Durrani Z. The inguinal paravascular technic of lumbar plexus anesthesia: the "3-in-1 block". Anesth Analg 1973; 52: 989-96.

9. Reavley P, Montgomery AA, Smith JE, Binks S, Edwards J, Elder G, et al. Randomised trial of the fascia iliaca block versus the ' 3 -in-1' block for femoral neck fractures in the emergency department. Emerg Med J 2015; 32: 685-9.

10. Salinas FV. Ultrasound and review of evidence for lower extremity peripheral nerve blocks. Reg Anesth Pain Med 2010; 35(2 Suppl): S16-25.

11. Cooper AL, Nagree Y, Goudie A, Watson PR, Arendts G. Ultrasound-guided femoral nerve blocks are not superior to ultrasound-guided fascia iliaca blocks for fractured neck of femur. Emerg Med Australas 2019; 31: 393-8.

12. Reid N, Stella J, Ryan M, Ragg M. Use of ultrasound to facilitate accurate femoral nerve block in the emergency department. Emerg Med Australas 2009; 21: 124-30.

13. Yun MJ, Kim YH, Han MK, Kim JH, Hwang JW, Do SH. Analgesia before a spinal block for femoral neck fracture: fascia iliaca compartment block. Acta Anaesthesiol Scand 2009; 53 : 1282-7.
14. Williams H, Paringe V, Shenoy S, Michaels P, Ramesh B. Standard preoperative analgesia with or without fascia iliaca compartment block for femoral neck fractures. J Orthop Surg (Hong Kong) 2016; 24: 31-5.

15. Groot L, Dijksman LM, Simons MP, Zwartsenburg MM, Rebel JR. Single fascia iliaca compartment block is safe and effective for emergency pain relief in hip-fracture patients. West J Emerg Med 2015; 16: 1188-93.

16. Fletcher AK, Rigby AS, Heyes FL. Three-in-one femoral nerve block as analgesia for fractured neck of femur in the emergency department: a randomized, controlled trial. Ann Emerg Med 2003; 41: 227-33.

17. Callear J, Shah K. Analgesia in hip fractures. Do fascia-iliac blocks make any difference? BMJ Qual Improv Rep 2016; 5: u210130.w4147.

18. Kumar D, Hooda S, Kiran S, Devi J. Analgesic efficacy of ultrasound guided FICB in patients with hip fracture. J Clin Diagn Res 2016; 10: UC13-6.

19. Ghimire A, Bhattarai B, Koirala S, Subedi A. Analgesia before performing subarachnoid block in the sitting position in patients with proximal femoral fracture: a comparison between fascia iliaca block and femoral nerve block. Kathmandu Univ Med J (KUMJ) 2015; 13: 152-5.

20. Capdevila X, Biboulet P, Bouregba M, Barthelet Y, Rubenovitch J, d'Athis F. Comparison of the three-in-one and fascia iliaca compartment blocks in adults: clinical and radiographic analysis. Anesth Analg 1998; 86: 1039-44.

21. Newman B, McCarthy L, Thomas PW, May P, Layzell M, Horn K. A comparison of pre-operative nerve stimulator-guided femoral nerve block and fascia iliaca compartment block in patients with a femoral neck fracture. Anaesthesia 2013; 68: 899-903.

22. Mather LE, Long GJ, Thomas J. The intravenous toxicity and clearance of bupivacaine in man. Clin Pharmacol Ther 1971; 12: 935-43. 